\title{
El farmacéutico en el contexto de las enfermedades raras y los medica- mentos huérfanos
}

\section{The pharmacist, rare diseases and orphan medicines}

\author{
C. Nagore, E. Lacalle, L. Arteche
}

\section{RESUMEN}

El tratamiento de las enfermedades raras muchas veces resulta complicado debido a las dificultades que plantean la investigación y desarrollo de medicamentos huérfanos por parte de la industria farmacéutica. Una de las mayores preocupaciones de este grupo de pacientes tan escaso y diverso al mismo tiempo, es el acceso a los fármacos disponibles.

La Agencia Europea del Medicamento establece la denominación de medicamentos huérfanos y los incentivos y bases necesarias para su comercialización.

La mayoría de los medicamentos huérfanos por sus especiales características, exigen una adecuada vigilancia y control. Los servicios de farmacia hospitalarios están activamente implicados en su gestión y dispensación, así como en un correcto seguimiento farmacoterapéutico de los pacientes.

Palabras clave. Medicamentos huérfanos. Enfermedades raras. Gestión. Atención farmacéutica.

An. Sist. Sanit. Navar. 2008; 31 (Supl. 2): 127-143.

\begin{abstract}
Treatment of rare diseases is often complicated due to the difficulties posed by the research and development of medicines by the pharmacological industry. One of the greatest concerns of this group of patients, which is both scarce and diverse, is access to available medicines.

The European Medicine Agency establishes the term orphan medicines and the incentives and bases for their commercialisation.

Due to their special characteristics, the majority of orphan medicines require suitable vigilance and control. The hospital pharmacy services are actively involved in their management and supply, as well as in a correct pharmacotherapeutic monitoring of the patients.
\end{abstract}

Key words. Orphan medicines. Rare diseases. Management. Pharmaceutical care.
Servicio de Farmacia. Hospital Virgen del Camino. Pamplona.

\section{Correspondencia:}

Camino Nagore Induráin

Servicio de Farmacia

Hospital Virgen del Camino

$\mathrm{C} /$ Irunlarrea $\mathrm{n}^{\circ} 4$

31008 Pamplona

Tfno. 848429453 


\section{INTRODUCCIÓN}

La Unión Europea (UE) define Enfermedades Raras (ER), como aquellas enfermedades incluidas las de origen genético, con peligro de muerte o de invalidez crónica, que tienen una prevalencia baja, menor de 5 casos por cada 10.000 habitantes en la Comunidad $^{1,2}$.

Bajo esta denominación se incluyen miles de enfermedades, sin embargo, individualmente presentan características muy dispares. El principal interés por agruparlas bajo la denominación de ER es conseguir aunar esfuerzos para fomentar la investigación y el interés de la sociedad por todas las ER en su conjunto.

En general, presentan muchas dificultades diagnósticas y de seguimiento, tienen un origen desconocido en la mayoría de los casos y conllevan múltiples problemas sociales. Además, dado que existen pocos datos epidemiológicos, plantean dificultades en la investigación debido a los pocos casos existentes. Por otra parte, si existen tratamientos efectivos, éstos no suelen ser económicamente rentables ${ }^{2}$.

La industria farmacéutica es reacia a invertir en el desarrollo de medicamentos (I+D) para tratamientos de enfermedades poco frecuentes por su escasa rentabilidad y alto costo de investigación y comercialización.

En todo este contexto, los pacientes con ER y sus familias se encuentran aislados, desinformados y enfrentados a nuevos problemas como el desconocimiento de tratamientos existentes, alternativas y forma de obtenerlos.

Las asociaciones de afectados de ER fueron quienes tomaron la iniciativa de defender el derecho a la salud de estos pacientes que carecían de tratamiento para su enfermedad, con el objeto de presionar a la Administración y a la sociedad para que ayudaran a paliar esta situación.

Fue Estados Unidos el primer país que desarrolló un texto legal en 1983, denominado The Orphan Drug Act, haciendo que el "estatus de huérfano" recibiera subvenciones para el desarrollo de estos productos, así como otras medidas de apoyo, hasta su aprobación comercial.
La UE inició una política común en este ámbito en 1999 . En diciembre de este mismo año el Parlamento Europeo y el Consejo aprobaron el Reglamento por el que se regulan los criterios para designar un medicamento como huérfano y propusieron establecer los incentivos para fomentar la investigación, el desarrollo y la comercialización de medicamentos destinados a prevenir, diagnosticar o tratar enfermedades poco frecuentes ${ }^{3}$.

Según la UE un medicamento huérfano $(\mathrm{MH})$ es aquel que cumple los siguientes requisitos:

1. Que se destine a establecer un diagnóstico, prevención o tratamiento de una enfermedad que afecte a menos de cinco personas por cada diez mil en la UE.

2. Que se destine al tratamiento de una enfermedad grave o incapacitante y cuya comercialización resulte poco probable (no comercial) sin medidas de estímulo.

3. Que sus beneficios sean significativos para los afectados ${ }^{3}$.

En el seno de la Agencia Europea del Medicamento (EMEA), en el año 2000 se crea el Comité de Medicamentos Huérfanos (COMP) y el Comité de Medicamentos para Uso Humano (CHMP). El primero es el responsable de la valoración científica que conduce a la designación de un medicamento como huérfano y el segundo es el responsable de la aprobación de su comercialización.

La Comisión de las Comunidades Europeas establece en abril de 2000 las disposiciones de aplicación de los criterios de declaración de los medicamentos huérfanos, y la definición de los conceptos de medicamento similar y superioridad clínica. Disposiciones que han de actualizarse periódicamente a la luz de los conocimientos científicos y técnicos y de la experiencia adquirida en la declaración y reglamentación de los medicamentos huérfanos (Diario Oficial L 103 de 28.4.2000) ${ }^{4}$.

Los $\mathrm{MH}$ tienen que someterse obligatoriamente a un procedimiento de registro centralizado de comercialización por el que se establecen procedimientos comuni- 
tarios para la autorización y el control de medicamentos de uso humano y veterinario $^{5}$.

El interés por estos MH y ER es un fenómeno relativamente nuevo en la mayor parte de los Estados miembros de la UE. En España se han organizado tres Congresos Internacionales de MH y ER (años 2000, 2004 y 2007), en estos eventos se pretende sensibilizar tanto a la sociedad en general como a las autoridades sanitarias, de las carencias y necesidades de los pacientes afectados por ER. Una de las preocupaciones planteadas ya en el «I Congreso Internacional de Enfermedades Raras y Medicamentos Huérfanos" celebrado en el año 2000 en Sevilla, fue el acceso de estos pacientes a los medicamentos disponibles mediante sistemas de distribución adecuados.

Esta problemática se refleja también en una nota de prensa de la Federación Española de Enfermedades Raras (FEDER) publicada recientemente con el título "El acceso a los medicamentos huérfanos para tratar las enfermedades raras, una asignatura pendiente en España"6.

A continuación se revisan los procedimientos de gestión de los MH. La mayoría son medicamentos extranjeros, no comercializados en España; otros están en fase de investigación clínica (usos compasivos y ensayos clínicos) y otros, por no estar disponibles en la preparación adecuada, deben prepararse como fórmulas magistrales en los servicios de farmacia.

La mayoría de los medicamentos designados como huérfanos y autorizados por la EMEA para su comercialización pertenecen a alguna de las categorías de "prescripción médica restringida" definidas en el artículo 24 del RD 1345/2007:

- Medicamentos de Uso Hospitalario (H): por sus características farmacológicas, su novedad o motivos de salud pública, se reservan para tratamientos que sólo puedan utilizarse o seguirse en medio hospitalario o centros asistenciales autorizados.

- Medicamentos de Diagnóstico Hospitalario de prescripción por determinados médicos especialistas (DH): se utilizan en el tratamiento de enfermedades que deben ser diagnosticadas en medio hospitalario, en establecimientos que dispongan de medios de diagnóstico adecuados o por determinados médicos especialistas, aunque la administración y seguimiento pueda realizarse fuera del hospital.

Por tanto, dadas las características especiales de los $\mathrm{MH}$, y cumpliendo la ley $29 / 2006$, de garantías y uso racional de los medicamentos y productos sanitarios que establece las funciones de los Servicios de Farmacia, el farmacéutico de hospital tiene un papel clave no sólo en términos burocráticos de gestión, sino también en la dispensación e información de medicamentos a todos los profesionales sanitarios ${ }^{8}$.

\section{GESTIÓN DE MEDICAMENTOS DE USO COMPASIVO}

Se entiende por uso compasivo (UC) de medicamentos la utilización en pacientes aislados, y al margen de un ensayo clínico, de medicamentos en fase de investigación, incluídas las especialidades farmacéuticas para indicaciones o condiciones de uso distintas de las autorizadas, cuando el médico bajo su exclusiva responsabilidad considera indispensable su utilización ${ }^{9}$.

En España el procedimiento de autorización, registro y condiciones de dispensación de los medicamentos de uso humano fabricados industrialmente se regula en el RD 1345/2007 , que deroga los artículos 28 y 29 del RD $223 / 2004^{9}$ en el que se define el UC, dejando en la actualidad un vacío legal en cuanto a la utilización y tramitación de solicitud del UC de medicamentos. En tanto se regulen éstos, la utilización de los medicamentos como UC se seguirá realizando conforme a las indicaciones contenidas en el RD 223/2004'.

Según la legislación actual, el UC de medicamentos sólo puede ser tramitado por un Servicio de Farmacia de hospital.

La solicitud de UC debe hacerse a la AEMPS dentro de la Dirección General de Farmacia y Productos Sanitarios (DGFPS), que procederá a autorizar o denegar cada solicitud particular. La denegación siempre irá acompañada de un informe justificando los motivos. 
La solicitud se acompañará de la siguiente documentación ${ }^{10}$ :

1. Consentimiento informado por escrito del paciente o de su representante legal en menores de edad e incapacitados. Los mayores de 12 años deberán también dar su consentimiento por escrito junto con el de su representante legal y será puesto en conocimiento del Ministerio Fiscal antes de empezar el tratamiento. El médico responsable del tratamiento será quien obtendrá el consentimiento informado del paciente. Para ello le entregará una hoja informativa con los detalles del mismo, su objetivo y beneficios esperados, riesgos y posibles efectos adversos, información sobre tratamientos alternativos disponibles (poco frecuente en ER) y sobre el carácter voluntario de la aceptación, protección de datos y compromiso de confidencialidad.

2. Informe clínico completo en el que el médico justifique la necesidad de dicho tratamiento.

3. La autorización del director del centro u hospital.

Una vez autorizado el UC por la AEMPS, el Servicio de Farmacia del hospital procederá a gestionar su adquisición:

- Si la especialidad farmacéutica está comercializada en España, se adquirirá del laboratorio nacional correspondiente.

- Si la especialidad farmacéutica es extranjera, el Servicio de Farmacia solicitará a la AEMPs la importación de la especialidad, indicando que es para un tratamiento de UC autorizado, ésta designará el laboratorio farmacéutico nacional o importador que lo suministrará al Servicio de Farmacia correspondiente.

La facturación de estos medicamentos irá a cargo del centro u hospital peticionario, y si éste es un centro del SNS, no se le facturará al enfermo el importe de la medicación.
La autorización de un medicamento como UC es válida para todo el tratamiento y para un paciente en concreto.

De acuerdo con la Normativa Europea, cada estado miembro es responsable de los tratamientos de uso compasivo que se realizan.

\section{GESTIÓN DE MEDICAMENTOS EXTRANJEROS}

El Ministerio de Sanidad y Consumo puede autorizar la importación de medicamentos legalmente comercializados en el extranjero y no autorizados en España, cuando resulte imprescindible para el tratamiento o diagnóstico de patologías concretas.

La AEMPS podrá conceder en situaciones específicas autorizaciones especiales supeditadas a la obligación por parte del solicitante de cumplir determinadas condiciones revisables anualmente ${ }^{8}$. La gestión de ME la tiene encomendada la DGFPS a través de la AEMPs.

A diferencia de la gestión de medicamentos de UC, que debe hacerse exclusivamente desde los Servicios de Farmacia de hospital, los ME pueden ser también tramitados a través de las entidades competentes de cada comunidad autónoma, para enfermos en régimen ambulatorio.

La documentación exigida depende del tipo de paciente y de la pertenencia o no al régimen de la Seguridad Social ${ }^{11}$ :

1. Informe del médico prescriptor que justifique la petición. El informe médico se renovará cuando lo exija el Ministerio de Sanidad y Consumo.

2. Documentos A-2 y A-3 (Anexos 1 y 2) en los que se especifican los datos del paciente y de la especialidad farmacéutica que se solicita. Sólo necesarios la primera vez que se solicita el medicamento para un paciente concreto. Los hospitales adjuntarán el modelo A-1 debidamente cumplimentado por el Servicio de Farmacia.

La importación de ME sólo se autorizará para las indicaciones aprobadas por las Autoridades Sanitarias del país de origen, 
de otro modo, se considerará a todos los efectos, investigación clínica y deberá ajustarse a lo establecido en la legislación actual sobre ensayos clínicos.

Si se autoriza la importación, el Ministerio de Sanidad y Consumo designará el laboratorio o a la empresa importadora que procederá a la importación y suministro al servicio solicitante. Si la solicitud es denegada, se comunica al centro peticionario, especificando el motivo de la misma, y éste a su vez lo comunicará al paciente y al médico, quien tras valorarla, podrá reiniciar los trámites, alegaciones, informes complementarios, etc., si procede.

La facturación sigue los mismos criterios que los vigentes para el resto de los medicamentos registrados en España.

En los hospitales del Sistema Nacional de Salud la dispensación la realiza el Servicio de Farmacia y el coste de la medicación va a cargo del hospital; en caso de ser privado lo facturará al paciente. Una de las preocupaciones de las asociaciones de pacientes es el de lograr sistemas de reembolso adecuado para pacientes que no puedan acceder al sistema público de salud, así como una equidad en el acceso y la calidad de los servicios sanitarios.

\section{ENSAYOS CLÍNICOS}

Una de las conclusiones recogidas en el "I Congreso de Enfermedades Raras y Medicamentos Huérfanos", es la de establecer créditos y ayudas públicas para la realización de ensayos clínicos (EC) con este tipo de fármacos.

Dada la baja prevalencia de ER y adicionalmente la extensa distribución geográfica de los afectados, el número de participantes en un EC suele ser reducido y, aunque la legislación no establece un mínimo de pacientes, en la práctica se considera cierto tamaño de muestra para asegurar la validez del ensayo según sea la fase del mismo, margen que en algunas fases es imposible cumplir con este tipo de enfermedades.

Una de las soluciones viables es la puesta en marcha de bases de datos de pacientes que permitan constituir grupos adecuados para realizar EC, así como para realizar la vigilancia postcomercialización.

Los EC con $\mathrm{MH}$ deben acogerse a la misma reglamentación que el resto de medicamentos:

- Las garantías de la investigación de los medicamentos de uso humano quedan recogidas en el titulo III (art. 58 a 62 ) de la Ley $29 / 2006^{8}$.

- La regulación de los EC con medicamentos se recoge en el RD 223/2004 de 6 febrero ${ }^{9}$.

En el caso de $\mathrm{MH}$, y cuando haya finalizado el plazo del EC, sólo podrá continuarse con la administración de los productos del ensayo (siempre que no estén autorizados para esas condiciones de uso) si se siguen las normas de UC, esto es una autorización temporal de utilización antes de la comercialización del fármaco (RD 223/2004, art. 29 actualmente derogado pero ante el vacío legal existente, sigue vigente) $)^{9}$.

En el caso de la población infantil, se deberían aplicar los requisitos de protección que se establecen para la realización de ensayos en pediatría. A pesar de que no se deberían extrapolar los resultados obtenidos en los EC realizados en adultos, muchas veces es difícil porque los estudios realizados en pediatría son escasos y a menudo se utilizan medicamentos que no tienen autorizada su indicación en esta población.

En el año 2006, el Parlamento y el Consejo Europeo aprobaron el Reglamento (CE) $\mathrm{n}^{\circ} 1901 / 2006$ sobre medicamentos para uso pediátrico ${ }^{12}$. Esta nueva reglamentación se adopta a raíz de una Resolución del Consejo en la que se invitaba a la Comisión a hallar soluciones al problema de la falta de medicamentos adecuados para los niños, tiene tres objetivos: garantizar que los medicamentos utilizados en pediatría sean objeto de una investigación de gran calidad; conseguir que paulatinamente estos medicamentos estén sujetos a una autorización adecuada; y velar por que pueda accederse a información de calidad sobre los medicamentos para uso pediátrico. 


\section{FORMULACIÓN MAGISTRAL}

La formulación magistral de $\mathrm{MH}$ es, sin duda alguna, una de las protagonistas en el proceso de utilización de estos fármacos personalizados. No debemos olvidar que una gran parte de estas enfermedades (alrededor del 50\%) afecta a la población pediátrica, por lo que una vez solucionada la adquisición del medicamento es muy probable que se necesiten dosificaciones o formas farmacéuticas distintas de la original, o bien que sea necesario acondicionar la materia prima para que se adapte mejor a las características del paciente.

Las fórmulas magistrales han de cumplir los criterios de eficacia y seguridad que establece el RD 175/2001 por el que se aprueban las normas de correcta elaboración y control de calidad de fórmulas magistrales y preparados oficinales ${ }^{13}$. Se elaborarán en las oficinas de farmacia y servicios farmacéuticos legalmente establecidos que dispongan de los medios necesarios para su preparación de acuerdo con las exigencias establecidas en el Formulario Nacional. Para la formulación magistral de sustancias o medicamentos no autorizados en España se requerirá el régimen previsto en el art 24 de la Ley $29 / 2006^{8}$. Este mismo RD, define la obligación de acompañar la dispensación con información pertinente y adecuada.

\section{EL FARMACÉUTICO DE HOSPITAL ANTE LA DISPENSACIÓN DE MEDICAMENTOS HUÉRFANOS}

Desde la Circular de 1991 de la Dirección General de Farmacia y Productos Sanitarios conocida como "Prosereme V"14, cada vez son más las especialidades farmacéuticas que se clasifican como "Uso Hospitalario". Estas especialidades, en cuyo material de acondicionamiento figura el símbolo "H" y que carecen de cupón precinto de la A.S.S.S, deben ser prescritas por un médico adscrito a los servicios de un hospital y dispensadas y controladas por los servicios de farmacia hospitalarios a los pacientes externos que estén en tratamiento.

Esta actividad es recogida por la Ley de Garantías y Uso Racional de los medica- mentos y productos sanitarios ${ }^{8}$, (y anteriormente la Ley 25/1990 del Medicamento), que establece que entre las funciones de un servicio de farmacia de hospital está la de "garantizar y asumir la responsabilidad técnica de la adquisición, calidad, correcta conservación, cobertura de las necesidades, custodia, preparación de fórmulas magistrales o preparados oficinales y dispensación de los medicamentos precisos para las actividades intrahospitalarias y de aquellos otros, para tratamientos extrahospitalarios, que requieran una particular vigilancia, supervisión y control."

La dispensación de medicamentos extrahospitalarios ha crecido espectacularmente en los últimos años derivando en la apertura de Consultas de Atención Farmacéutica a pacientes externos (CAF). Las CAF son actualmente estructuras consolidadas orgánicamente dentro del servicio de farmacia hospitalario; y funcionalmente, con asistencia reconocida, están incluidas dentro de la cartera básica de servicios.

Las CAF tienen como objetivo general la dispensación de medicamentos y material sanitario, estableciendo un adecuado seguimiento en cada caso y desarrollando técnicas de educación sanitaria e información sobre los tratamientos y productos dispensados. Asimismo promueven la utilización racional de los medicamentos y/o productos sanitarios que deban ser dispensados, siempre de acuerdo a la legislación vigente, de ámbito estatal o autonómico, que le sea de aplicación con el fin $\mathrm{de}^{15}$ :

- Conseguir la adherencia al mismo.

- Garantizar la correcta conservación de los medicamentos.

- Informar sobre el tratamiento farmacológico al paciente.

- Detectar posibles incidencias o problemas relacionados con el medicamento.

- Evitar los errores asociados al uso de los medicamentos.

- Establecer el seguimiento farmacoterapéutico al paciente. 
- Articular la comunicación e intercambio de información entre el farmacéutico y el equipo asistencial.

- Optimizar los recursos sanitarios disponibles.

Sin embargo, en las CAF no se dispensan únicamente las especialidades farmacéuticas de Uso Hospitalario, también son susceptibles de ser dispensados los medicamentos de uso compasivo, ensayos clínicos, algunos medicamentos extranjeros, y todas aquellas especialidades o indicaciones que, sin pertenecer a estas categorías, las autoridades sanitarias determinen. En estas categorías, como se ha comentado anteriormente, se incluyen la mayoría de los medicamentos huérfanos.

El "Consenso sobre Atención Farmacéutica" publicado por el Ministerio de Sanidad y Consumo en $2002^{16}$, define la Atención Farmacéutica (AF) como "la participación activa del farmacéutico para la asistencia al paciente en la dispensación y seguimiento de un tratamiento farmacoterapéutico, cooperando así con el médico y otros profesionales sanitarios a fin de conseguir resultados que mejoren la calidad de vida del paciente".

Son actividades de AF realizadas en las CAF, la prevención de la enfermedad, educación sanitaria, farmacovigilancia y el seguimiento farmacoterapéutico. Este término es definido como la práctica en la que el farmacéutico se responsabiliza de las necesidades del paciente relacionadas con los medicamentos. Esto se realiza mediante la detección de Problemas Relacionados con la Medicación (PRM) y resolución de Resultados Negativos asociados a la Medicación (RNM). Este servicio implica un compromiso, y debe proveerse de forma continuada, sistematizada y documentada, en colaboración con el propio paciente y con los demás profesionales del sistema de salud, con el fin de alcanzar resultados concretos que mejoren la calidad de vida del paciente ${ }^{17}$.

En el momento que un médico especialista quiere prescribir un medicamento de dispensación hospitalaria, se pone en contacto con el Servicio de Farmacia de su hospital a través de un informe médico. El farmacéutico estudia la solicitud y realiza las gestiones oportunas para disponer del mismo (tramitación de UC, medicamentos extranjeros, pedidos a los laboratorios proveedores, entre otros). Una vez que el medicamento está en la farmacia, se avisa al paciente para su recogida en la consulta de AF.

En la primera visita, el farmacéutico informa sobre el medicamento que debe tomar el paciente, haciendo hincapié en la posología, forma de administración, efectos adversos y modo de conservación del mismo. Generalmente estas explicaciones van acompañadas de información escrita, para que el paciente pueda recurrir a ella si lo necesita (Anexo 3). No hay que olvidar que los prospectos de los medicamentos extranjeros no suelen estar en español. Además de dispensar la medicación correspondiente a 1-3 meses de tratamiento (dependiendo de la política del hospital), el farmacéutico entrega al paciente una tarjeta de citación en la que se anota la fecha de la próxima visita, intentando adaptarla a las preferencias del paciente o a su consulta médica. En esta tarjeta, figura también el teléfono de la consulta, al que puede recurrir para aclarar cualquier duda relacionada con el tratamiento. El anexo 4 muestra un ejemplo de tarjeta de citación.

En las sucesivas visitas, el farmacéutico realizará un seguimiento fármaco-terapéutico del paciente, en el cual mediante una entrevista clínica estructurada, comprobará la asimilación de la información, tratará de detectar PRM y estimará el grado de adherencia al tratamiento. Si se estima oportuno, se reforzará la información sobre los medicamentos con el objetivo de conseguir un uso adecuado de los mismos. Todas estas actividades deben ser registradas para un correcto seguimiento del paciente, y ante cualquier incidencia, se contactará con el médico especialista para mantenerle informado.

En el caso de los $\mathrm{MH}$, estas actividades de AF son de gran importancia. La escasa experiencia clínica que se dispone con estos tratamientos unida a la baja prevalencia de estas enfermedades hace que todos los profesionales de la salud debamos estar alerta ante posibles reacciones 
adversas que puedan aparecer y no hayan sido descritas. En este sentido, el RD $711 / 2002$, de 19 de julio, por el que se regula la farmacovigilancia de medicamentos de uso humano, establece la obligación de los profesionales sanitarios de colaborar con el Sistema Español de Farmacovigilancia, mediante la notificación al centro de referencia de cada comunidad autónoma de las sospechas de reacciones adversas a medicamentos no descritas o de gran importancia.

\section{Situación en la Comunidad Foral de Navarra}

De los $44 \mathrm{MH}$ comercializados por la EMEA, hasta la fecha actual, los que se están utilizando o han sido utilizados en los distintos hospitales del Servicio Navarro de Salud-Osasunbidea son 18.

La tabla 1 intenta ser un reflejo de las principales características de estos medicamentos: indicaciones, posología y aspectos a tener en cuenta. 
EL FARMACÉUTICO EN EL CONTEXTO DE LAS ENFERMEDADES RARAS Y LOS MEDICAMENTOS HUÉRFANOS

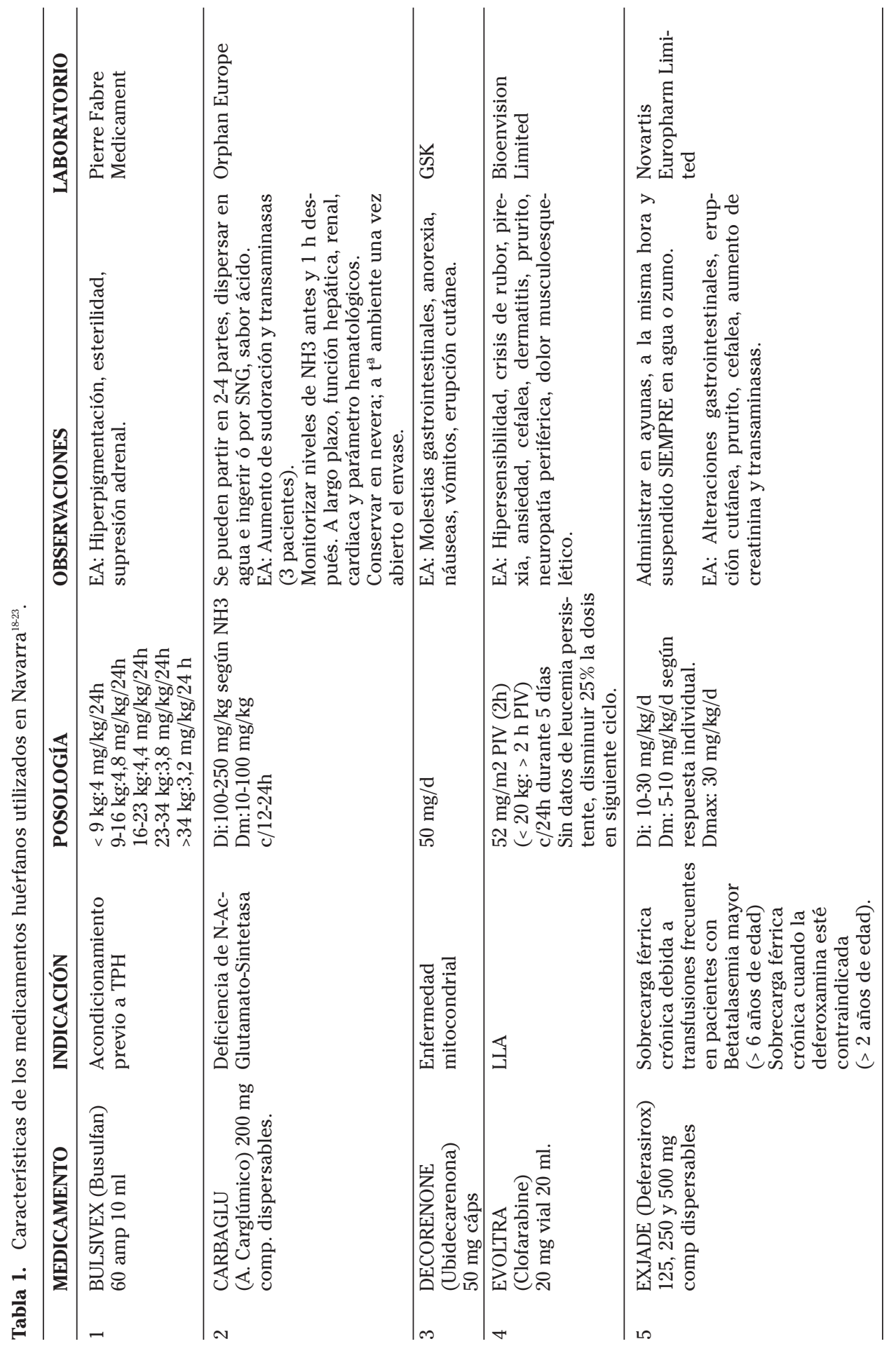




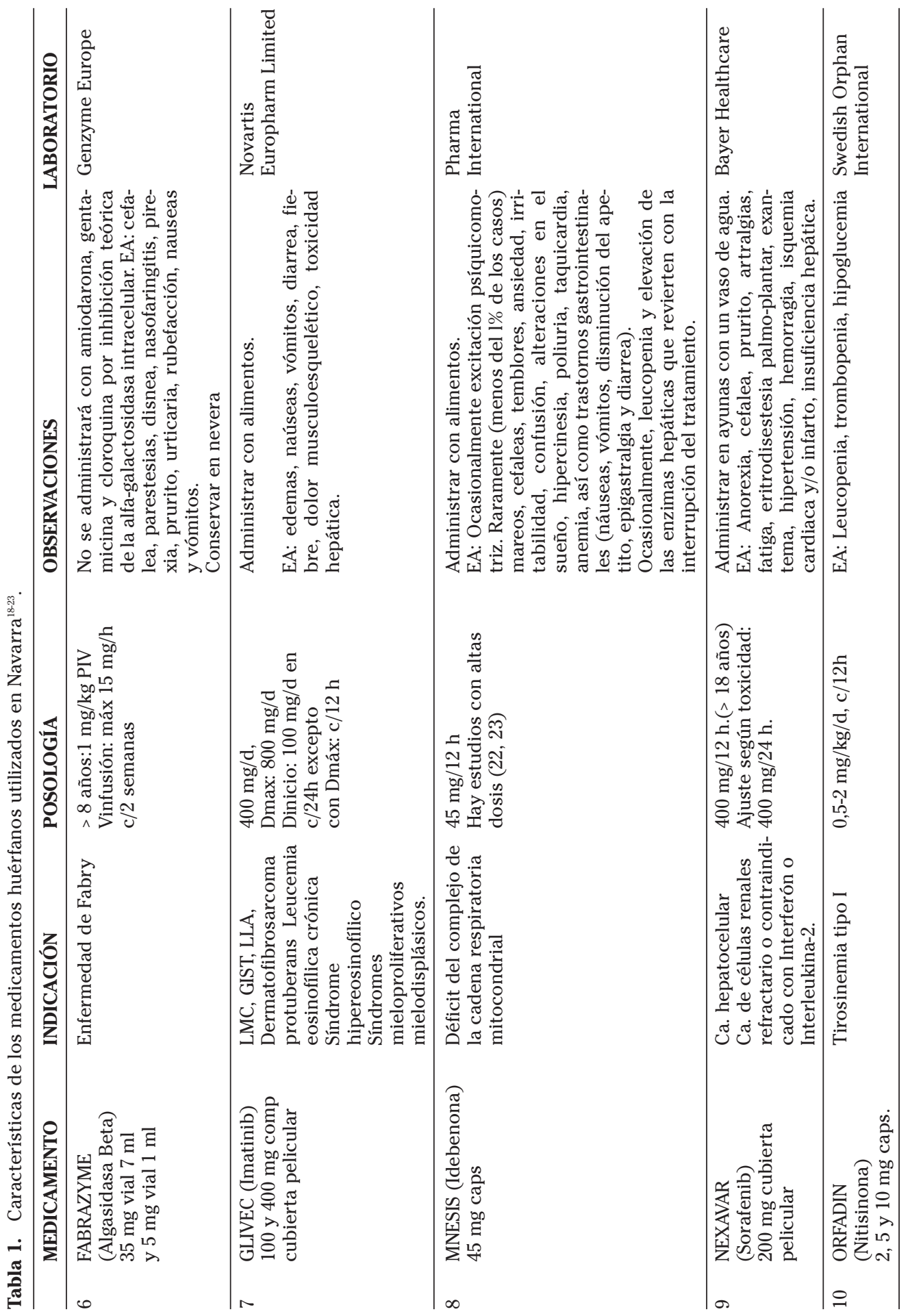


EL FARMACÉUTICO EN EL CONTEXTO DE LAS ENFERMEDADES RARAS Y LOS MEDICAMENTOS HUÉRFANOS

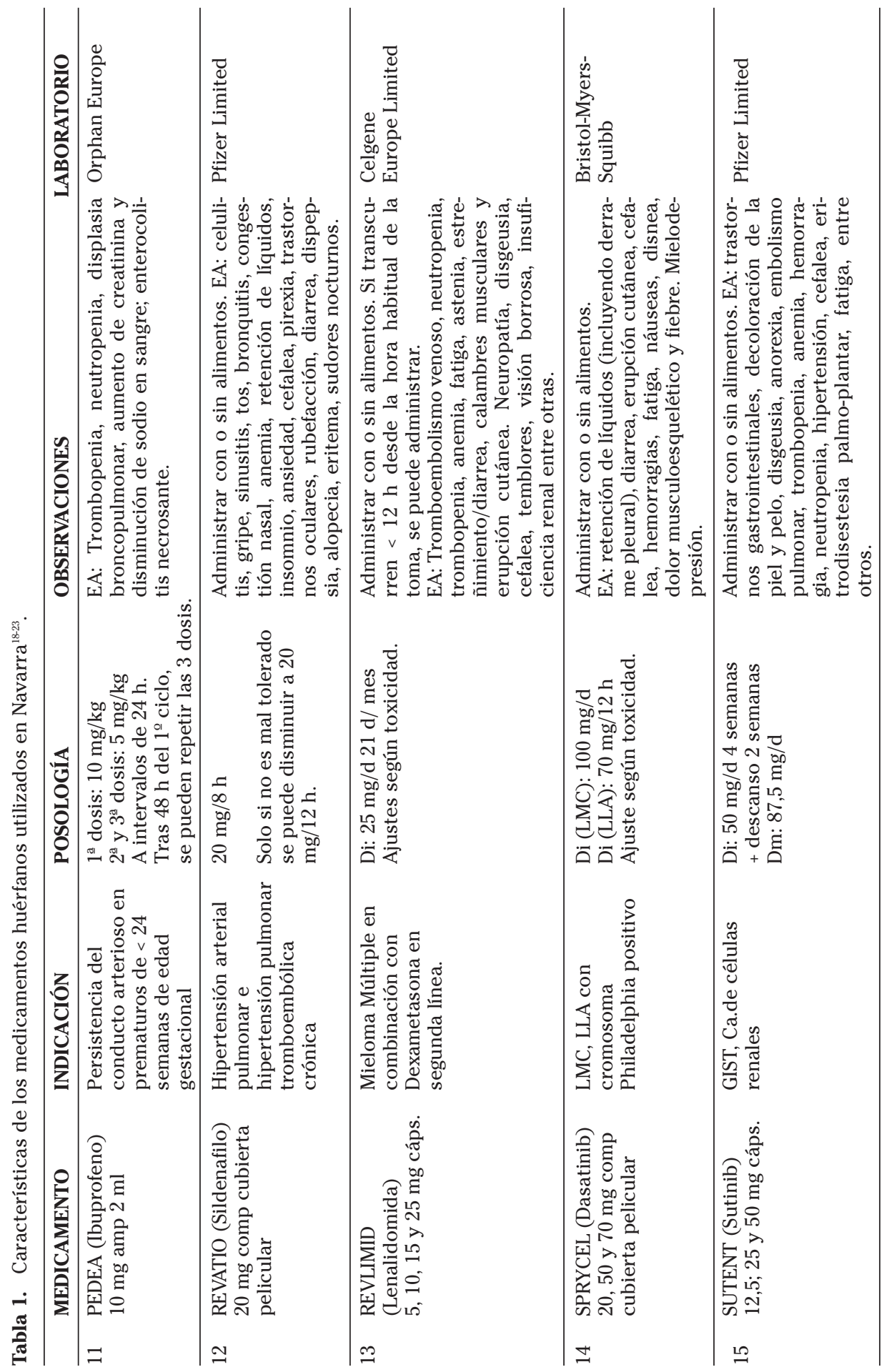




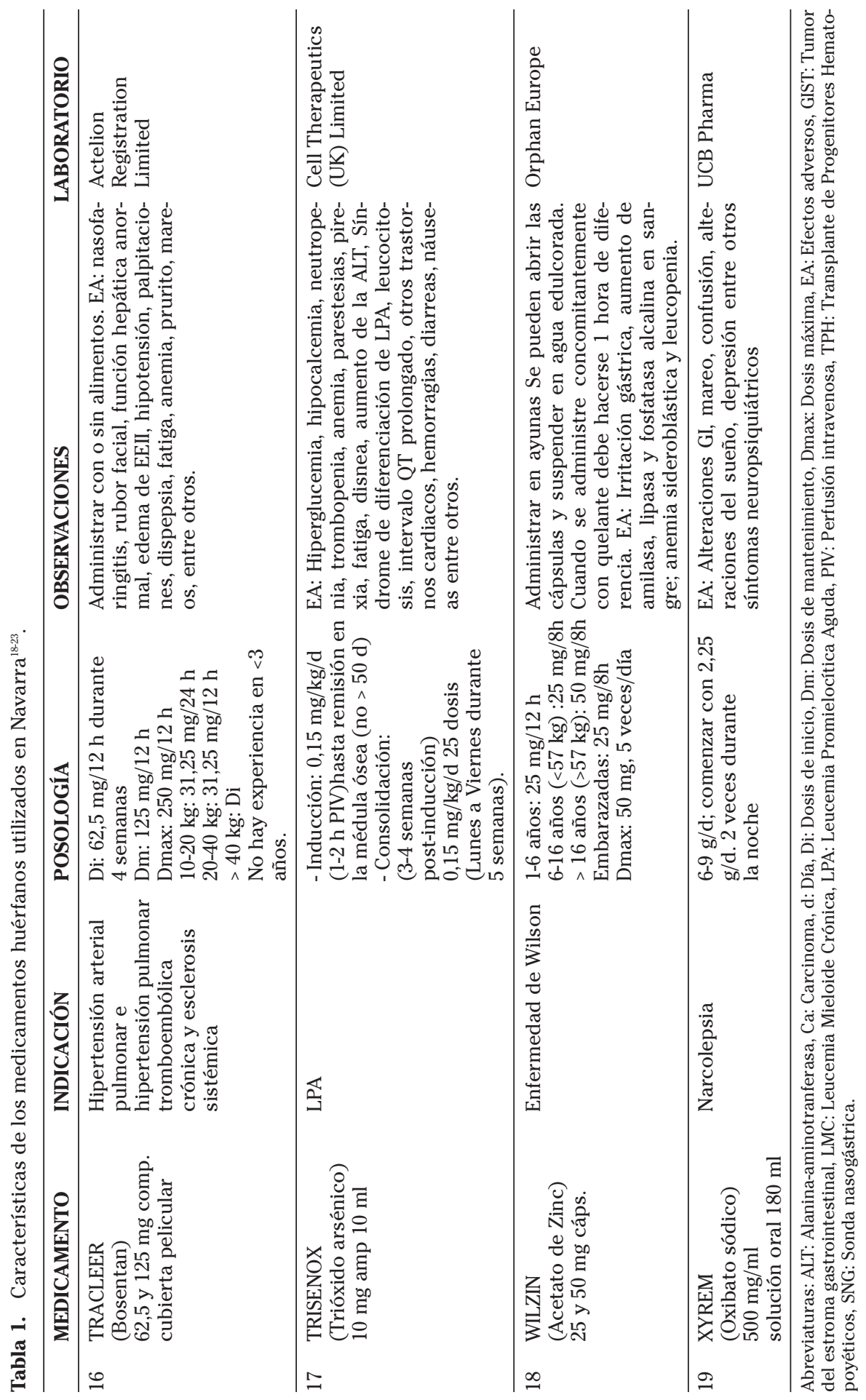




\section{BIBLIOGRAFÍA}

1.

http://ec.europa.eu/health/ph_threats/non _com/rare_diseases_es.htm

2. http://iier.isciii.es/er/html/er_faqs.htm

3. Reglamento (CE) $\mathrm{n}^{\mathrm{o}} 141 / 2000$ del Parlamento Europeo y del Consejo, de 16 de diciembre de 1999, sobre medicamentos huérfanos.

4. Reglamento (CE) $\mathrm{n}^{\mathrm{o}} 847 / 2000$ de la Comisión, de 27 de abril de 2000, por el que se establecen las disposiciones de aplicación de los criterios de declaración de los medicamentos huérfanos y la definición de los conceptos de "medicamento similar» y "superioridad clínica»

5. Reglamento (CE) No 726/2004 del Parlamento Europeo y del Consejode 31 de marzo de 2004, DOCE del 30 de abril, por el que se establecen procedimientos comunitarios para la autorización y el control de los medicamentos de uso humano y veterinario y por el que se crea la Agencia Europea de Medicamentos.

6. Nota de prensa de 26/03/08. El acceso a los medicamentos huérfanos para tratar las Enfermedades Raras, una asignatura pendiente en España. http://www.discapnet.es/Discapnet/Castell ano/Actualidad/Nueva_Hemeroteca/detalle ?id=185423

7. Real Decreto 1345/2007, de 11 de octubre, por el que se regula el procedimiento de autorización, registro y condiciones de dispensación de los medicamentos de uso humano fabricados industrialmente. BOE $\mathrm{n}^{\mathrm{o}}$ 267, de 7 de noviembre de 2007.

8. Ley $29 / 2006$, de 26 de julio, de garantías y uso racional de los medicamentos y productos sanitarios. BOE $\mathrm{n}^{\circ} 178$, de 27 de julio de 2006.

9. Real Decreto $223 / 2004$ de 6 febrero, BOE del 7 , que regula los ensayos clínicos con medicamentos. BOE $\mathrm{n}^{\mathrm{0}} 33$, de 7 de febrero de 2004.

10. http://www.ub.es/legmh/erpcompa.htm

11. http://www.ub.es/legmh/erpmedex.htm
12. Reglamento (CE) $\mathrm{n}^{\mathrm{o}}$ 1901/2006 del Parlamento Europeo y del Consejo sobre medicamentos para uso pediátrico.

13. Real Decreto $175 / 2001$, de 23 de febrero, por el que se aprueban las normas de correcta elaboración y control de calidad de fórmulas magistrales y preparados oficinales. BOE $\mathrm{n}^{\circ} 65$, de 16 de marzo de 2001.

14. Circular 11/91 de la Dirección de Farmacia y Productos Sanitarios del Ministerio de Sanidad y Consumo, que establece el Procedimiento Selectivo de Revisión de Medicamentos V (PROSEREME V).

15. Normas de Dispensación a Pacientes Externos. Recomendaciones de la SEFH para el desarrollo de Atención Farmacéutica a pacientes externos con dispensación.

16. Grupo de Consenso. Documento de Consenso en Atención Farmacéutica. Ministerio de Sanidad y Consumo. Ars Pharm 2001; 42: 223-243.

17. Panel de consenso. Tercer Consenso de Granada sobre Problemas Relacionados con Medicamentos y Resultados Negativos asociados a la Medicacion. Ars Pharm 2007; 48: 5-17.

18. Orphanet Reports Series- List of marketing authorised Orphan drugs in Europe. January 2008.

19. http://www.orpha.net/orphacom/cahiers/docs/GB/List_of_marketing_orphan _drugs_in_Europe.p

20. Fichas técnicas según la EMEA: http://www.emea.europa.eu_

21. Federación española de enfermedades raras (FEDER) http://www.enfermedadesraras.org/

22. Di Prospero NA, BAKer A, JeFFries N, FischBeck KH. Neurological effects of high-dose idebenone in patients with Friedreich's ataxia: a randomised, placebo-controlled trial. Lancet Neurol 2007; 6: 878-886.

23. Di Prospero NA, Summer CJ, PenzaK SR, Ravina B, FischBECK KH, TAYLOR JP. Safety, tolerability and pharmacokinetics of highdose idebenone in patients with Friedreich ataxia. Arch Neurol 2007; 64: 803-808. 
Anexo 1. Impreso A2 de medicamentos extranjeros.

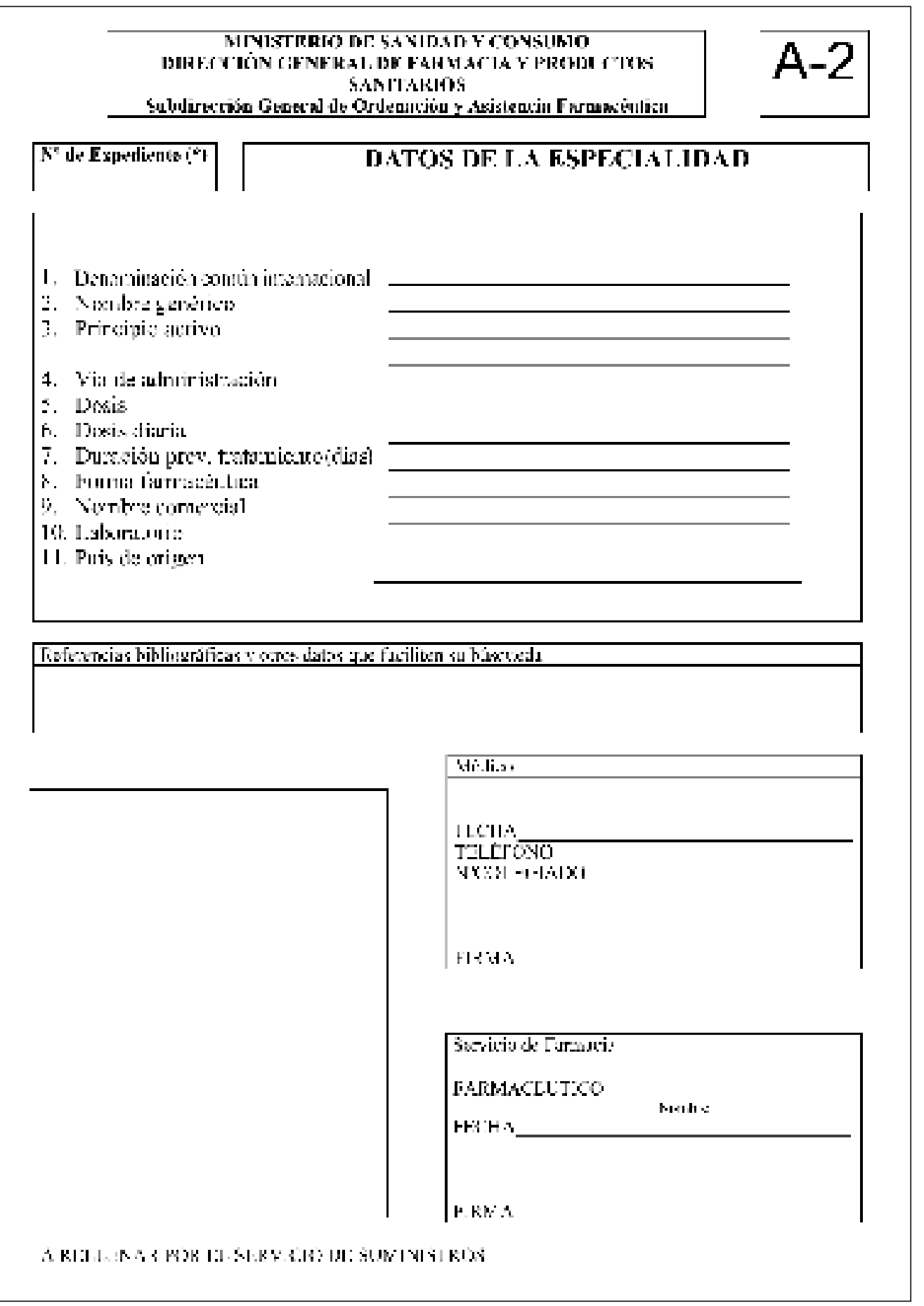


Anexo 2. Impreso A3 de medicamentos extranjeros.

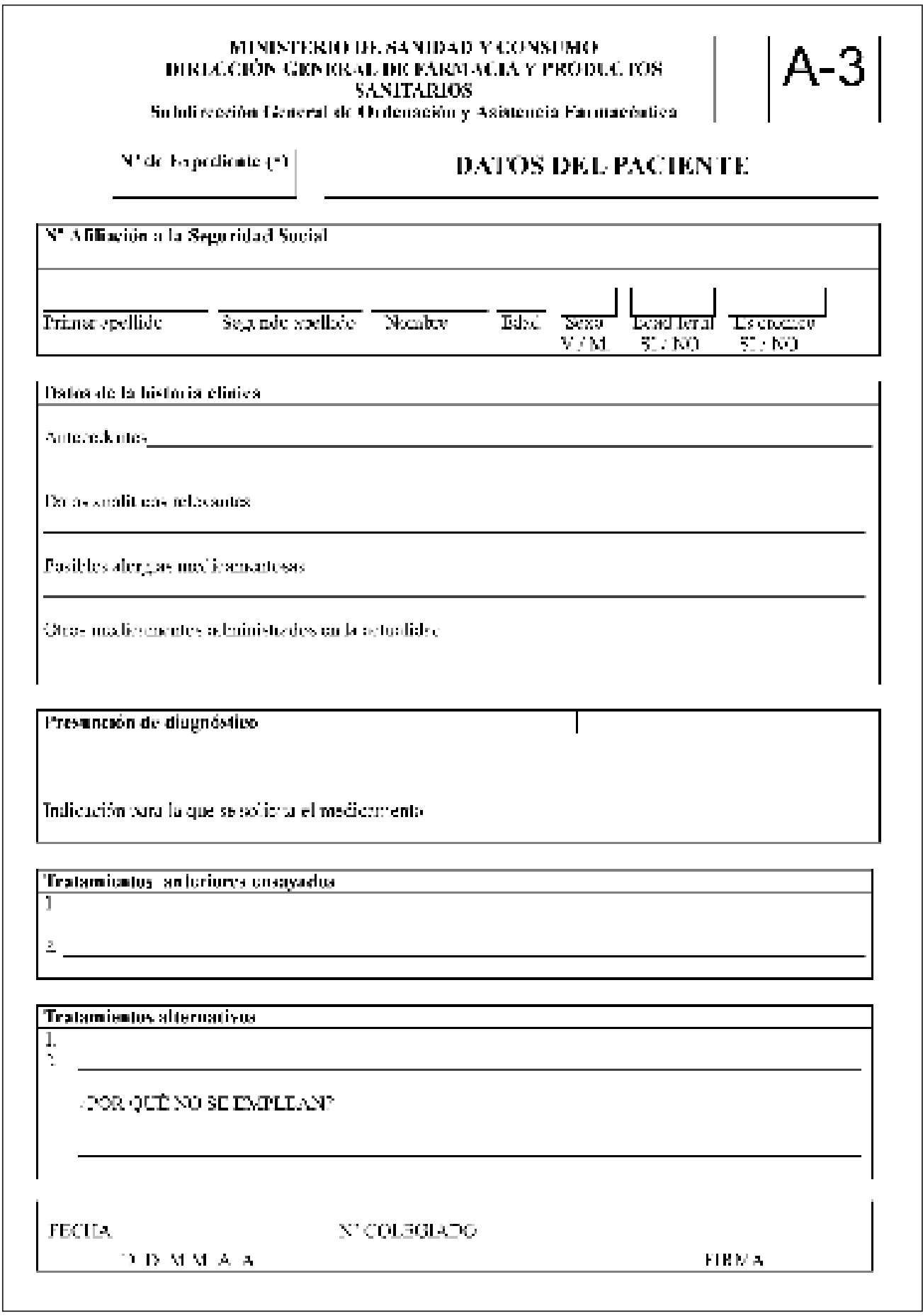


Anexo 3. Ejemplo de hoja de información escrita para el pacientes.

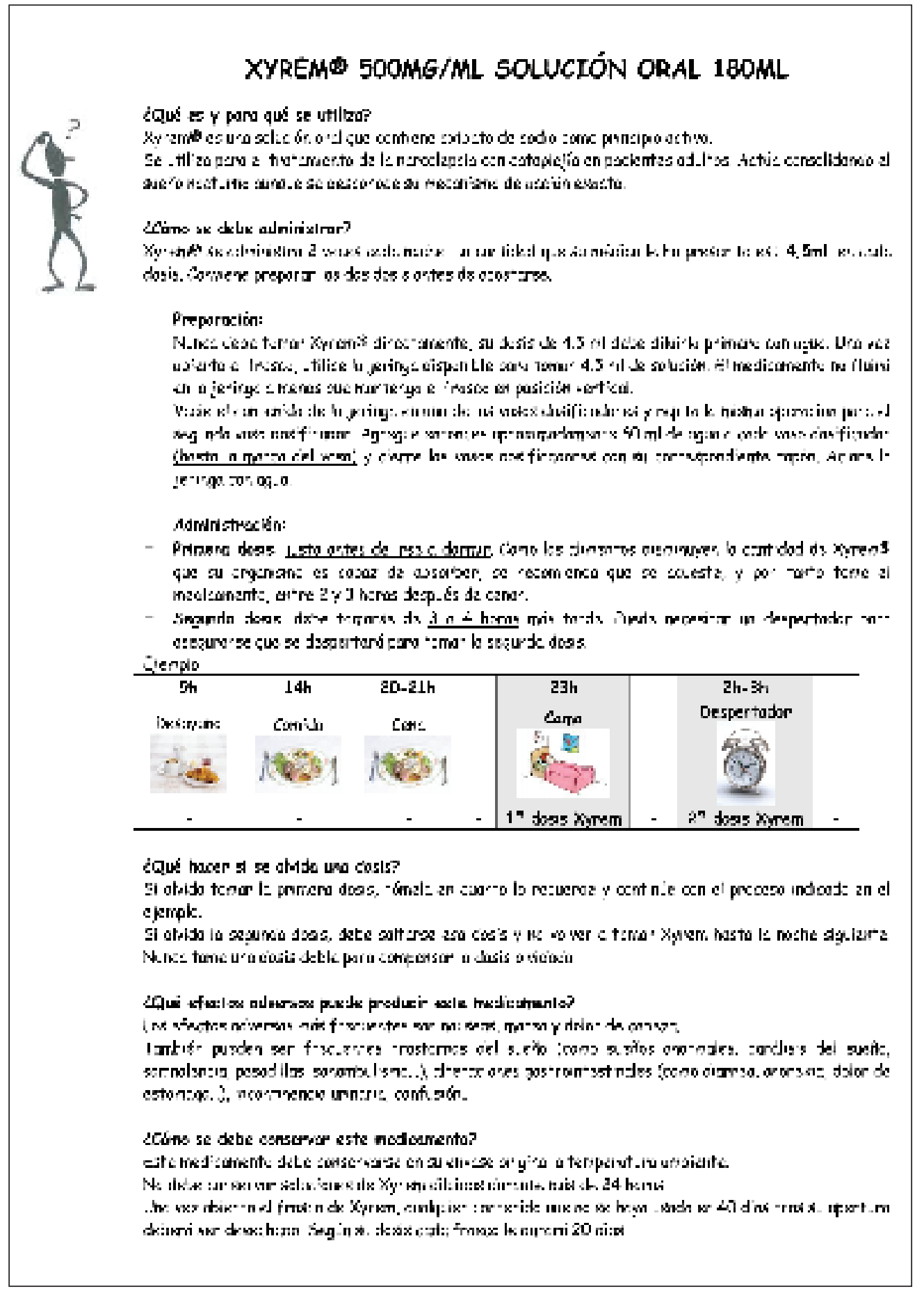


Anexo 4. Tarjeta de citación de la consulta de atención farmacéutica del HVC.

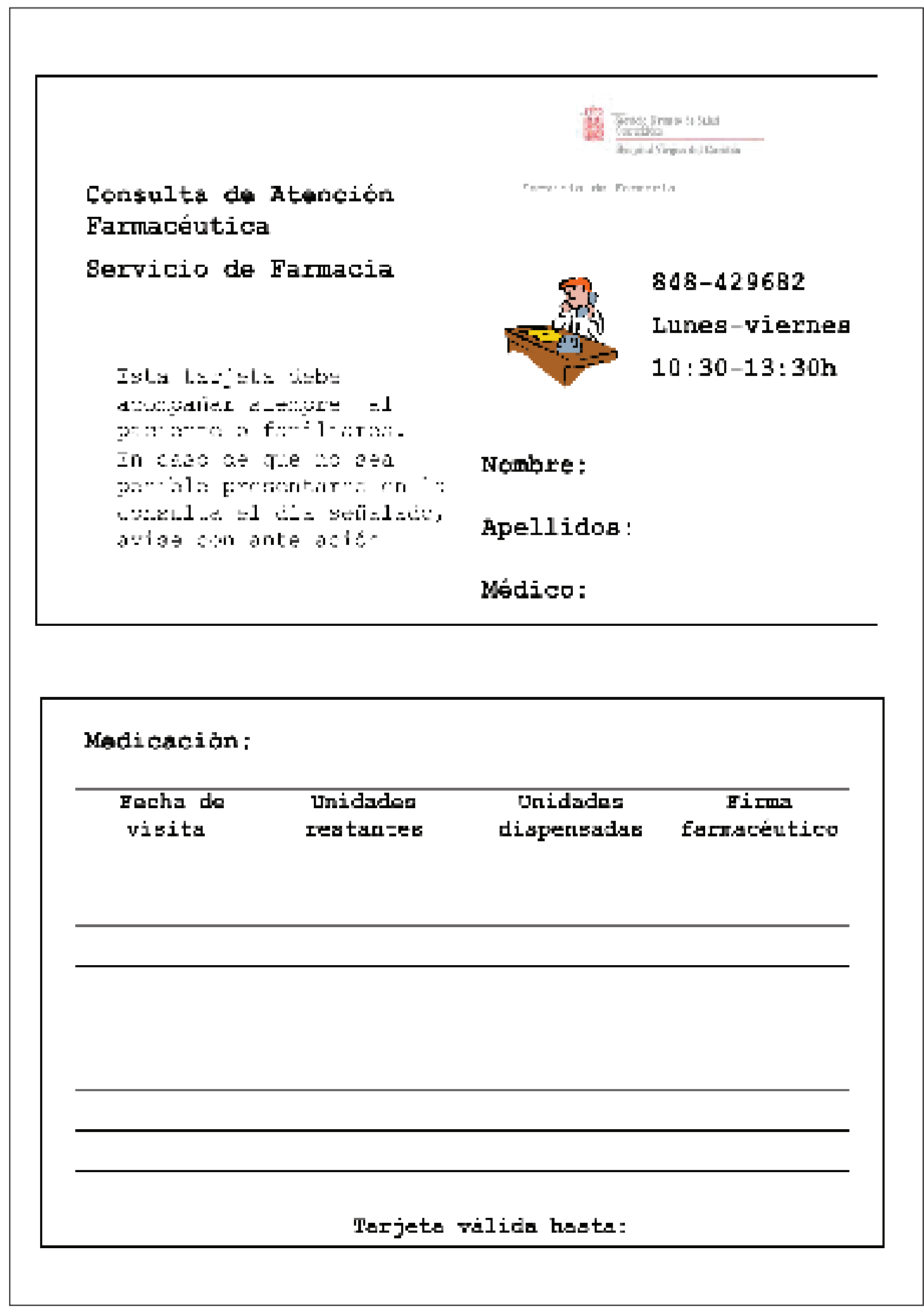


